\title{
Analysis of the Human Role in Planning and Scheduling via System Dynamics
}

\author{
Katsumi Morikawa and Katsuhiko Takahashi \\ Graduate School of Engineering, Hiroshima University, \\ 1-4-1, Kagamiyama, Higashi-Hiroshima 739-8527, Japan \\ TEL: +81-82-424-7704, FAX: +81-82-422-7024 \\ \{mkatsumi, takahasi\}@hiroshima-u.ac.jp
}

\begin{abstract}
A system dynamics model is proposed to analyze the human role in planning and scheduling. Based on the interview research results planning and scheduling activities in manufacturing companies are investigated. By selecting important elements in planning and scheduling, a model of the human role is developed assuming a simple manufacturing environment. Two modes of the model is examined in the simulation experiments, and the results indicate the importance of the human role in smoothing the workload by lookahead decisions and in reducing the uncertainty by collecting information and coordinating planning and scheduling activities.
\end{abstract}

Keywords

Planning, scheduling, human, system dynamics

\section{Introduction}

Manufacturing companies have been facing with the world-wide severe competition. Selecting and introducing a suitable integrated production planning and management system is an important strategic decision to improve the productivity and cost competitiveness. Although many companies have already introduced such planning systems successfully, we found that introducing an entirely new planning system without understanding manufacturing conditions and current roles of the human planner and scheduler may lead to unsatisfactory consequences [1]. Most manufacturing organizations still require human contributions (Jackson et al. [2]), and much research effort has been devoted to investigating difficulties in real-life planning and scheduling tasks (for example, Stoop and Wiers [3]), and analyzing/ 
modeling their activities (Crawford et al. [4], MacCarthy et al. [5], and MacCarthy and Wilson [6]). These studies emphasize the importance of human contributions, but such importance is not recognized properly in some companies we have investigated. Qualitative and quantitative researches are still required to develop and introduce successful production planning systems that work with humans collaboratively.

The aim of this study is to propose a model of production planning and scheduling activities of humans, and analyze the human role through simulation experiments. In making the model it is difficult to select detailed elements and then formulate quantitative equations between them. Therefore this study focuses on macro-level elements and develops the model using system dynamics. Detail of the model is explained after discussing the planning and scheduling activities considered in this study.

\section{Production Planning and Scheduling}

Defining the meaning of planning and scheduling is not so simple. McKay and Wiers [7] have classified the tasks of planner, scheduler, and dispatcher based on a long period of investigation of companies. This study implicitly assumes several small or medium scale companies interviewed [1] and define the planner and scheduler, and their tasks as follows.

The planner decides daily production plans often using the production planning system. The production plan generally defines the product types to be produced, the production quantities of these products, and due dates of them. The planner cannot always use the production plan produced by the production planning system because of the following reasons; (i) the planning system cannot consider all of the capacity constraints of the shop floor in general, (ii) information within the planning system is sometimes different from the actual conditions, (iii) the planning system cannot handle some information such as expected future orders appropriately. However, except for making very simple products, the information system is an important tool that can process complicated information effectively. Thus the planner generates production plan by obtaining necessary information from the shop floor and the sales department, by using interactive planning/re-planning function of the system, and finally by modifying the obtained plan manually if such modifications are needed.

Based on the upper planner's decision, the scheduler decides detailed production schedules. Typical decisions include worker allocation, machine allocation, and job sequencing. These decisions often need a deep knowledge about the shop floor conditions and also the processing requirements of orders. Therefore, a foreman often plays the role of the scheduler. In make-to-order companies producing different products everyday, it is very difficult to estimate the workload of each order acccurately. The foreman often can give good estimation based on his (her) long experience. When making detailed schedules, sophisticated optimization techniques are seldom used in practice. Instead, the foreman often makes schedules based on simple calculations using spread sheet software and his (her) experience. 
The human role fairly depends on the manufacturing environments. The planner of companies producing a limited number of product types repetitively by assembling parts with relatively simple operations can produce detailed production plans using the production planning system. In such companies, the human role is mainly to handle unexpected events such as machine failures, delivery delay, and so on. On the other hand, if the processing time of operations inevitably fluctuates, or planned production lead time is fairly long and thus it is often required to modify the plans, the automatic planning is difficult and thus the adjustment of plans by the human planner and scheduler must be important.

\section{A Model of the Human Role via System Dynamics}

In this section we propose a model of the human role in planning and scheduling based on system dynamics. Several authors have already proposed several production planning and inventory control models via system dynamics. Two examples are a domestic manufacturing company illustrated by Coyle [8], and a supply chain model of Intel by Gonçalves et al. [9]. Although these models are highly helpful in developing our model, this study has focused on the conditiondependent actions of the planner and scheduler, and the collaborative decisionmaking between them at a deeper level. To our limited knowledge, there seems no similar system dynamics-based approach for modeling the planner and scheduler.

Fig. 1 shows the developed causal loop of the decision activities of planner and scheduler under a hypothetical manufacturing environment based on the activities described in section 2. The upper area of the figure corresponds to the planner's decisions, and the lower area to the scheduler's decisions. The boundary of these two areas is not rigid and may depend on manufacturing environments. Needless to say actual planners and schedulers consider many other factors and make several other decisions. The objective of the proposed causal loop is not to provide a comprehensive model, but rather to focus on typical decision making activities and illustrate their relations.

The primal task of the production planner is to select items to be produced and decide their production quantities. In the proposed model all orders are measured in hours, and the planner decides the order release rate $(R R)$ of the received orders $(R O)$. The system normally needs a fixed order process time $(D 1)$ to release the received orders. Therefore, the feasible release rate of orders is $R O / D 1$. However, the planner also considers other factors. First is the workload of the shop floor (WL). If the current workload of the shop floor is relatively high, it may be better to reduce the release rate based on the level of the current workload. The model assumes that the desired workload $(D W L)$ of the shop floor is given by the regular production time $(R E G)$ multiplied by the planned lead time ( $P L T)$. If the shop floor is overloaded, i.e., $W L>D W L$, and the feasible release rate is greater than the regular production capacity, it may be better to reduce the release rate. On the other hand, if the feasible release rate is less than the regular production capacity, and the workload of the shop floor is less than the desired level, the planner may go to the sales department and collect the information about expected future orders. Let $E O$ denote the workload of 
the expected orders of the next working day. If $E O$ is greater than the regular production capacity, a candidate look-ahead procedure is to expedite required processes for the current received orders and to prepare for the expected future orders immediately. These activities can increase the feasible release rate to some extent, and thus it is expected to reduce the future overtime production. Based on the above idea, the release rate is defined by the following equation:

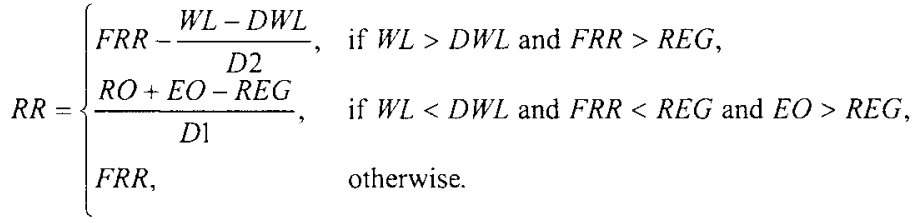

where $D 2$ means a constant value representing the length of time to resolve the overload.

The planned lead time is a coordination parameter between the planner and scheduler. We assume that both the planner and scheduler know the possible shortest lead time $(S L T)$. However the shortest lead time often incurs difficulties in making detailed schedules within the regular production time. If the planned lead time is longer than the shortest time, the scheduler can make the following decisions; reduce the number of set up operations by combining the same or similar orders, adjust the processing sequence to improve the equipment utilization, allocate orders to workers considering the skill level of each worker, and so on. The degree of freedom of scheduler's decision increases with the increase of planned lead time. This relation is expressed by the efficiency term in the figure because we assume that the planner can produce efficient schedules using the planning freedom. The actual production time is largely determined by the production rate and efficiency of the schedule, but partially affected by the uncertainty involved in the environment. If the actual overtime production time is relatively large when compared with the expected time, the scheduler requests the increase of the planned lead time to hedge against the uncertainty. However, selecting a long planned lead time beyond a certain level is unacceptable from the planner's viewpoint because of higher work-in-process inventory and longer response to customer demand. Therefore deciding the best planned lead time considering these conflicting factors is an important role of the planner and scheduler.

The decision of production rate $(P R)$ is the primal task of the scheduler. A normal production rate $(N P R)$ can be defined as follows: $N P R=W L / P L T$. In some manufacturing environments, the scheduler has a certain degree of freedom in deciding the production rate. For example, the scheduler may be able to add workload of 30 minutes in addition to $N P R$ by making a good schedule, or shift a partial amount of workload to the next working day. This type of freedom is named adjustable range $(A R)$ in Fig. 1. For example, $A R=5 \%$ means the scheduler can adjust the workload up to $0.05 \cdot R E G$. Fig. 1 also shows the information flow from order release rate $(R R)$ to production rate $(P R)$. If the information exchange between the planner and scheduler is conducted properly, the scheduler can know the current release rate in addition to the current workload level. Based on the above discussions, the production rate is assumed to be defined as follows: 


$$
P R= \begin{cases}R E G, & \text { if } N P R \geq(1-A R) \times R E G \text { and } N P R<R E G \text { and } R R>R E G, \\ R E G, & \text { if } N P R \leq(1+A R) \times R E G \text { and } N P R>R E G \text { and } R R<R E G, \\ N P R, & \text { otherwise. }\end{cases}
$$

The meaning of the above equation is straightforward. For example, the top equation means that if the normal production rate is slightly lower than the regular production capacity, and the release rate is increasing, then increase the production rate to the regular production time. Such decisions are expected to reduce the cumulative overtime production.

A remaining important equation is the following balance equation between the inflow, stock, and outflow of workload:

$$
\frac{d W L}{d t}=R R-P R
$$

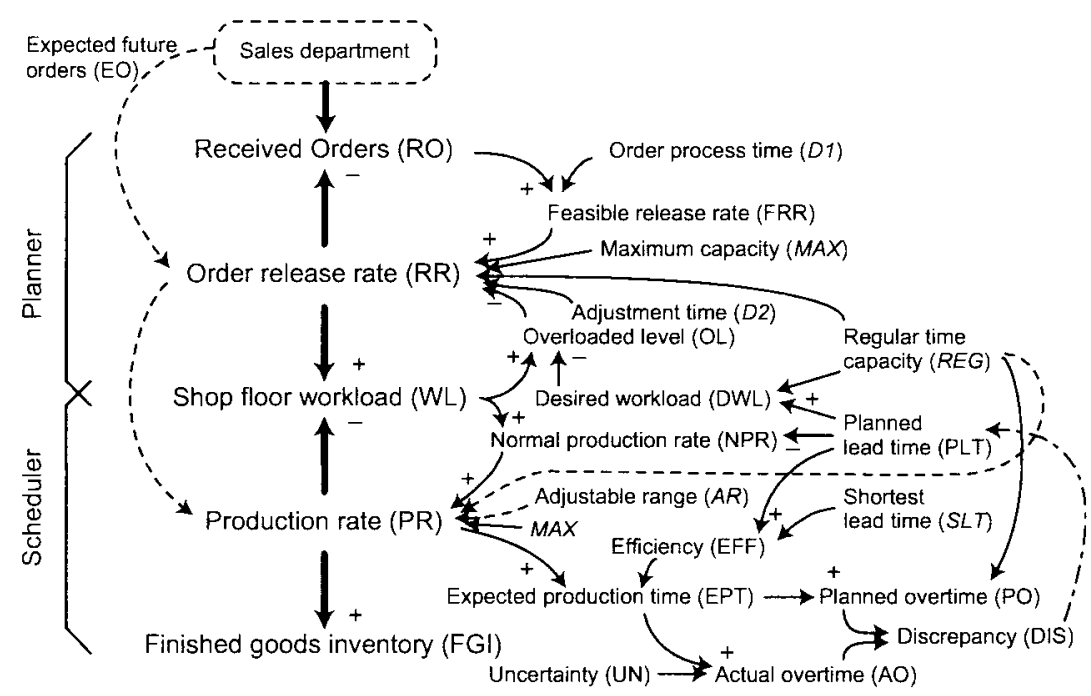

Fig. 1. Causal loop diagram of planning and scheduling activities

\section{Simulation Experiments}

Based on the causal loop diagram shown in Fig. 1, two modes of the simulation model were developed; simple mode and full mode. The simple mode was composed of elements shown in Fig. 1 except the relationships shown in broken lines. On the other hand, the full mode included all relationships shown in Fig. 1. However, both modes excluded the directed broken arc started from DIS (discrepancy) to PLT (planned lead time). This relation was considered separately in the simulation experiments. 
The following values of the input parameters were used in all simulation runs: $R E G=8$ (hours), $M A X=10$ (hours), $D 1=4$ (days), $D 2=3$ (days), $S L T=5$ (days). Fluctuating demand was generated randomly within the interval $[5,12]$ (hours) in each day. Three adjustable ranges $(A R), 0 \%, 5 \%$, and 10\%, were prepared, and the level of uncertainty was selected from the following values; $0 \%, 2.5 \%, 5 \%, 10 \%$, $15 \%$, and $20 \%$. When the planned lead time (PLT) was 5 days, there was no efficiency improvement. If $P L T$ was 5.5 or 6 , the efficiency improvement was $2.5 \%$ or $5 \%$, respectively. The length of the simulation was 250 working days, and all random events were generated using the same seed in all simulation runs.

The primal performance measure is the cumulative overtime production in hours. Utilizing the available regular production time as much as possible, and avoid the overtime production if possible is often considered as an important planning goal. Fig. 2 shows the cumulative overtime of the simple and full modes under several uncertainty levels and adjustable ranges when the planned lead time was 5.5 (upper graphs) or 6 (lower graphs). As we assumed that longer planned lead time enables higher production efficiency, the cumulative overtime increased when the planned lead time was 5.5. We can interpret the figure as follows: (i) Increasing the level of uncertainty increased the cumulative overtime production. (ii) The full mode was effective in reducing overtime production when compared with the simple mode. (iii) In full mode, increasing the adjustable range also reduced the cumulative overtime. (iv) The difference of cumulative overtime between the simple mode and the full mode with $A R=0 \%$ expresses the effectiveness of releasing workload considering the future expected orders. The results indicate that the planner's look-ahead releasing decision reduced the cumulative overtime. (v) When the planned lead time (PLT) was 5.5 and thus the efficiency was $2.5 \%$, the differences in overtime between two modes and between different adjustable ranges in full mode were both larger when compared with the results of $P L T=6$. This means that the full mode is an effective model in utilizing the limited available capacities. In other words, decisions of planner and scheduler highly affect the cumulative overtime production especially under higher workload conditions. Fig. 2 also indicates that the performance of the simple and full modes was relatively stable when the level of uncertainty was small. This means that the importance of the planner and scheduler is enhanced when the shop floor condition is relatively unstable and uncertain. Namely, reducing the uncertainty by collecting up-to-date information and coordinating planning and scheduling activities is an important role of the planner and scheduler.

The average workload level is also an important performance measure when deciding the planned lead time. Fig. 3 shows the effect of planned lead time on the cumulative overtime and the average shop floor workload. Bold lines correspond to the full mode, and thin lines to the simple mode. As the average workload was nearly the same for both modes, one broken line is used in the figure. We assumed that the production efficiency increases when the planned lead time is increased. Therefore the cumulative overtime decreased for increased planned lead time. On the other hand, the average shop floor workload increased, and the rate of increase was proportional to the regular time capacity. This behavior can be explained by the structure of the causal loop model shown in Fig. 1. Another important role of the planner and scheduler is to adjust the planned lead time adequately by considering the trade-off between the average workload and the expected overtime. Although the 
proposed model does not have an explicit function to describe this type of decision, the importance of selecting the appropriate length of the planned lead time is partially explained by the simulation results.

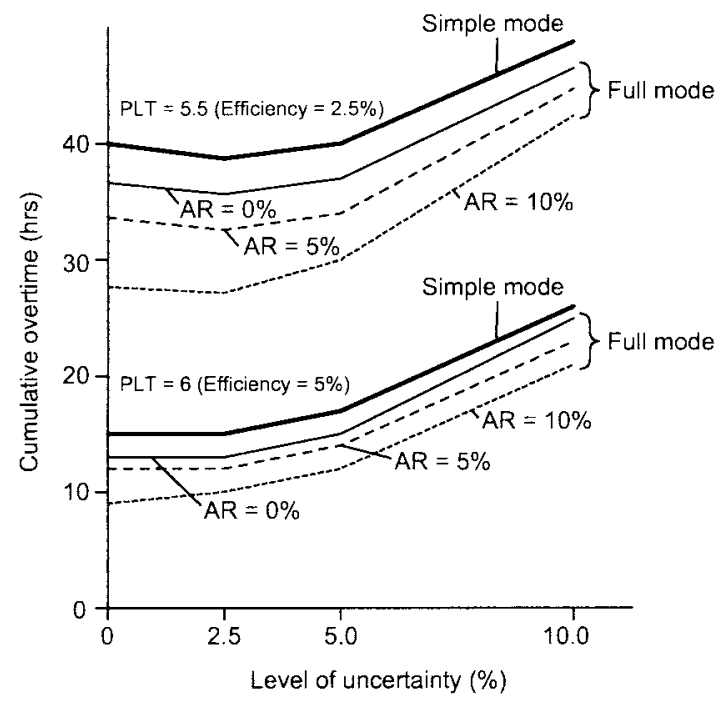

Fig. 2. The cumulative overtime of simple and full modes

\section{Conclusion}

This paper describes planning and scheduling activities in manufacturing companies based on the interview research conducted previously, and then proposes a system dynamics-based model of the human role in planning and scheduling. The proposed model involves the condition-dependent actions of the planner and scheduler, and the collaborative decision-making between them at a deeper level. Simulation experiments using two modes of the proposed model illustrate the importance of the human role in smoothing the workload by look-ahead decisions and in lowering the uncertainty by effective information exchange. Enhancing the model considering other important activities is needed in future study. In addition, validation and refinement of the model based on actual data is also an important remaining work.

\section{Ackowledgment}

This research was partially supported by the Japan Society for the Promotion of Science with a Grant-in-Aid for Scientific Research, No. 19510149. 


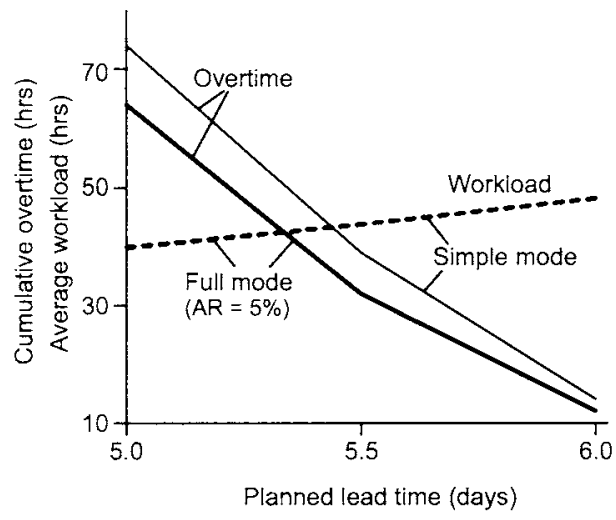

Fig. 3. The cumulative overtime and average workload varying the planned lead time when $U N=2.5 \%$

\section{References}

1. K. Morikawa and K. Takahashi, Modeling planning and scheduling tasks based on interviews, Proceedings of the 7th Asia Pacific Industrial Engineering \& Management Systems Conference, December 17-20, Bangkok, Thailand, 16571661 (2006).

2. S. Jackson, J. R. Wilson, and B. L. MacCarthy, A new model of scheduling in manufacturing: Tasks, roles, and monitoring, Human Factors, 46(3), 533-550 (2004).

3. P. P. M. Stoop and V. C. S. Wiers, The complexity of scheduling in practice, International Journal of Operations \& Production Management, 16(10), 37-53 (1996).

4. S. Crawford, B. L. MacCarthy, J. R. Wilson and C. Vernon, Investigating the work of industrial schedulers through field study, Cognition, Technology \& Work, 1(2), 63-77 (1999).

5. B. L. MacCarthy, J. R. Wilson, and S. Crawford, Human performance in industrial scheduling: A framework for understanding, Human Factors and Ergonomics in Manufacturing, 11(4), 299-320 (2001).

6. B. L. MacCarthy and J. R. Wilson (Editors), Human Performance in Planning and Scheduling (Taylor \& Francis, London, 2001).

7. K. N. McKay and V. C. S. Wiers, Planning, scheduling and dispatching tasks in production control, Cognition, Technology \& Work, 5(2), 82-93 (2003).

8. R. G. Coyle, System Dynamics Modelling: A Practical Approach (Chapman \& Hall, London, 1996).

9. P. Gonçalves, J. Hines, and J. Sterman, The impact of endogenous demand on push-pull production systems, System Dynamics Review, 21(3), 187-216 (2005). 